\title{
HUA'S INEQUALITY FOR COMPLEX NUMBER
}

\author{
SEVER SILVE'יRU DRAGOMIR
}

Abstract. Variants for the complex numbers of the celebrated Lo-Keng Hua's inequality which is very important in Number Theory are given.

1. The following inequality due to Lo-Keng Hua is important in Number Theory [1]:

$$
\left(\delta-\sum_{i=1}^{n} x_{i}\right)^{2}+\alpha \sum_{i=1}^{n} x_{i}^{2} \geq k_{n} \delta^{2},
$$

where $\delta, \alpha>0, x_{i} \in \mathbb{R}(i=\overline{1, n})$ and $k_{n}=\alpha(n+\alpha)^{-1}$ with equality if and only if $x_{i}=h_{n} \delta$ where $h_{n}=(n+\alpha)^{-1}$.

Recently, Chung-Lie Wang [2] gave the following interesting generalization of (1):

Theorem A. Let $\alpha, \delta$ be as above. Then for $p>1$, the inequality

$$
\left(\delta-\sum_{i=1}^{n} x_{i}\right)^{p}+\alpha^{p-1} \sum_{i=1}^{n} x_{i}^{p} \geq k_{n}^{p-1} \delta^{p}
$$

holds for all nonnegative $x_{i} \in \mathbb{R}(i=\overline{1, n})$ with $\sum_{i=1}^{n} x_{i} \geq \delta$. The sign of inequality in (2) is reversed for $0<p<1$. In either case, the sign of equality holds in (2) iff $x_{i}=h_{n} \delta(i=\overline{1, n})$.

An intergral variant is also given.

2. In this paper, we shall give two variants of inequality (1) for complex numbers.

The following lemma holds

Lemma. Let $\alpha>0$ and $\delta, z \in \mathbb{C}$. Then the following inequality

$$
|\delta-z|^{2}+\alpha|z|^{2} \geq \frac{d|\delta|^{2}}{1+\alpha}
$$

Received February 23, 1994; revised June 6, 1994.

1991 Mathematics Subject Classification. 26D15.

Key words and phrases. Hua's inequality, complex numbers. 
holds. The equality is valid in (3) iff

$$
z=\frac{1}{1+\alpha} \delta .
$$

Proof. If $\delta=0$, the inequality in (3) is obvious.

Suppose that $\delta \neq 0$. Then by the inequality $|\delta-z| \geq|| \delta|-| z||$ and by Hua's result for $n=1$ we have

$$
|\delta-z|^{2}+\alpha|z|^{2} \geq(|\delta|-|z|)^{2}+\alpha|z|^{2} \geq \frac{\alpha|\delta|^{2}}{1+\alpha} .
$$

The second equality in (4) holds (see Hua's result for $n=1$ ) iff

$$
|z|=\frac{1}{1+\alpha}|\delta| .
$$

On the other hand, the first equality is valid in (4) iff

$$
|\delta-z|^{2}=|| \delta|-| z||^{2}, \quad \text { i.e., } \quad \operatorname{Re}(\bar{\delta} \cdot z)=|\delta||z| .
$$

Suppose that $z=\lambda \delta$ with $\lambda \in \mathbb{C}$. Then by (6) we have

$$
|\delta|^{2} \operatorname{Re}(\lambda)=|\lambda||\delta|^{2}
$$

and, since $\delta \neq 0$, we deduce

$$
\operatorname{Re}(\lambda)=|\lambda|=\left([\operatorname{Re}(\lambda)]^{2}+[\operatorname{Im}(\lambda)]^{2}\right)^{1 / 2}
$$

i.e., $\lambda \in \mathbb{R}$ and moreover, $\lambda>0$, hence by the equality (5) we deduce

$$
\lambda|\delta|=\frac{1}{\alpha+1}|\delta|
$$

which give us $\lambda=\frac{1}{\alpha+1}$.

Consequently the equality holds in (3) iff $z=\frac{1}{\alpha+1} \delta$.

We can give the following variant of Hua's inequality for complex numbers.

Theorem 1. Let $\alpha>0$ and $\delta, z_{1}, \ldots, z_{n} \in \mathbb{C}$. Then the following inequality

$$
\left|\delta-\sum_{i=1}^{n} z_{i}\right|^{2}+\alpha \sum_{i=1}^{n}\left|z_{i}\right|^{2} \geq \frac{\alpha|\delta|^{2}}{n+\alpha}
$$

holds. The equality is valid in (7) iff:

$$
z_{i}=\frac{\delta}{n+\alpha} \quad(i=\overline{1, n}) .
$$


Proof. By the well known Cauchy-Buniakowski-Schwarz's inequality for complex numbers:

$$
n \sum_{i=1}^{n}\left|z_{i}\right|^{2} \geq\left|\sum_{i=1}^{n} z_{i}\right|^{2}
$$

with equality iff $z_{i}=\lambda \in \mathbb{C}$ for all $i \in\{1, \ldots, n\}$, one has

$$
\left|\delta-\sum_{i=1}^{n} z_{i}\right|^{2}+\alpha \sum_{i=1}^{n}\left|z_{i}\right|^{2} \geq\left|\delta-\sum_{i=1}^{n} z_{i}\right|^{2}+\frac{\alpha}{n}\left|\sum_{i=1}^{n} z_{i}\right|^{2} .
$$

Now, using the above lemma, we have:

$$
\left|\delta-\sum_{i=1}^{n} z_{i}\right|^{2}+\frac{\alpha}{n}\left|\sum_{i=1}^{n} z_{i}\right|^{2} \geq \frac{\alpha|\delta|^{2}}{n+\alpha}
$$

with equality iff

$$
\sum_{i=1}^{n} z_{i}=\frac{n}{n+\alpha} \delta
$$

Consequently, the equality holds in (7) iff

$$
n \lambda=\sum_{i=1}^{n} z_{i}=\frac{n}{n+\alpha} \delta
$$

i.e., $\lambda=\frac{\delta}{n+\alpha}=z_{i}$ for all $i \in\{i, \ldots, n\}$, and the proof is finished.

3. The following generalization of inequality (7) also holds.

Theorem 2. Let $\alpha>0$ and $\delta, z_{i}, w_{i} \in \mathbb{C}(i=\overline{1, n})$. Then the following inequality:

$$
\left|\delta-\sum_{i=1}^{n} z_{i} w_{i}\right|^{2}+\alpha \sum_{i=1}^{n}\left|z_{i}\right|^{2} \geq \frac{\alpha|\delta|^{2}}{\alpha+\sum_{i=1}^{n}\left|w_{i}\right|^{2}}
$$

holds. The equality is true in (8) iff

$$
z_{i}=\frac{\delta \bar{w}_{i}}{\alpha+\sum_{i=1}^{n}\left|w_{i}\right|^{2}} \quad \text { for all } \quad i \in\{1, \ldots, n\} .
$$

Proof. If $\sum_{i=1}^{n}\left|w_{i}\right|^{2}=0$, i.e., $w_{i}=0$ for all $i \in\{1, \ldots, n\}$, the inequality (8) is valid.

Suppose that $\sum_{i=1}^{n}\left|w_{i}\right|^{2}>0$, then by Cauchy-Buniakowski-Schwarz's inequality

$$
\left|\sum_{i=1}^{n} z_{i} w_{i}\right|^{2} \leq \sum_{i=1}^{n}\left|z_{i}\right|^{2} \sum_{i=1}^{n}\left|w_{i}\right|^{2}
$$


with equality iff $z_{i}=\lambda \bar{w}_{i}(\lambda \in \mathbb{C})$ for all $i \in\{1, \ldots, n\}$, we have

$$
\left|\delta-\sum_{i=1}^{n} z_{i} w_{i}\right|^{2}+\alpha \sum_{i=1}^{n}\left|z_{i}\right|^{2} \geq\left|\delta-\sum_{i=1}^{n} z_{i} w_{i}\right|^{2}+\frac{\alpha}{\sum_{i=1}^{n}\left|w_{i}\right|^{2}}\left|\sum_{i=1}^{n} z_{i} w_{i}\right|^{2} .
$$

Now, using the above lemma, we get:

$$
\left|\delta-\sum_{i=1}^{n} z_{i} w_{i}\right|^{2}+\frac{\alpha}{\sum_{i=1}^{n}\left|w_{i}\right|^{2}}\left|\sum_{i=1}^{n} z_{i} w_{i}\right|^{2} \geq \frac{\alpha|\delta|^{2}}{\alpha+\sum_{i=1}^{n}\left|w_{i}\right|^{2}}
$$

with equality iff

$$
\sum_{i=1}^{n} z_{i} w_{i}=\frac{\sum_{i=1}^{n}\left|w_{i}\right|^{2}}{\alpha+\sum_{i=1}^{n}\left|w_{i}\right|^{2}} \delta
$$

But $z_{i}=\lambda \bar{w}_{i}(i=\overline{1, n})$ hence the equality holds in (8) iff

$$
z_{i}=\lambda \bar{w}_{i}=\frac{\delta \bar{w}_{i}}{\alpha+\sum_{i=1}^{n}\left|w_{i}\right|^{2}} \quad(i=\overline{1, n})
$$

and the proof is finished.

Remark 1. If in (8) we put $w_{i}=1(i=\overline{1, n})$ we recapture (7).

Remark 2. ILet $\alpha>0$ and $\delta, z_{i}, w_{i} \in \mathbb{C}(i=\overline{1, n})$ with $\left|w_{i}\right|=1$ for all $i \in$ $\{1, \ldots, n\}$. Then one has the inequality

$$
\left|\delta-\sum_{i=1}^{n} z_{i} w_{i}\right|^{2}+\alpha \sum_{i=1}^{n}\left|z_{i}\right|^{2} \geq \frac{\alpha|\delta|^{2}}{\alpha+n}
$$

with equality iff

$$
z_{i}=\frac{\delta \bar{w}_{i}}{n+\alpha}, \quad i \in\{1, \ldots, n\}
$$

i.e., a class of Hua's type inequalities for complex numbers.

\section{References}

[1] L. K. Hua, "Additive Theory of Prime Numbers (Translated by N. B. Ng) in Translations of Math. Monographs," Vol. 13, Amer. Math. Soc. Providence, RI, 1965.

[2] C.-L. Wang, Lo-Keng Hua, "Inequality and dynamic programming," J. Math. Anal. Appl., 166 (1992), 345-350.

Department of Mathematics, University of Timişoara, B-dul V. Pârvan 4, RO-1900 Timişoara, România. 